\title{
Crystal Structure of Streptozocin
}

\author{
Masayuki Haramura, * Akito Tanaka,* Toshio Aкimoto, ${ }^{* *}$ and Noriaki Hirayama**† \\ *Reverse Proteomics Research Institute Co. Ltd., 2-6-7 Kazusa-Kamatari, Kisarazu, Chiba 292-0818, Japan \\ **Basic Medical Science and Molecular Medicine, Tokai University School of Medicine, \\ Boseidai, Isehara, Kanagawa 259-1193, Japan
}

\begin{abstract}
The crystal belongs to space group $P 22_{1} 2_{1} 2_{1}$, and the cell dimensions are $a=8.5300(5), b=11.4516(9)$ and $c=$ 23.893(1) A. The final $R$ value is 0.047 . There are two crystallographically independent molecules in an asymmetric unit. The pyranose rings in both molecules take chair conformations. The nitrosourea moieties are planar. There is an extensive hydrogen network in the crystal.
\end{abstract}

(Received August 8, 2003; Accepted October 27, 2003; Published on Web December 17, 2003)

Streptozocin (2-deoxy-2-[[(methylnitrosoamino)carbonyl]amino]D-glucopyranose)(Fig. 1) is an antibiotic derived from Streptomyces acromogenes. This naturally occurring nitrosourea is useful in the treatment of human pancreatic islet cell carcinoma and malignant carcinoid tumors. It also has a high affinity for $\beta$ cells of the islets of Langerhans and causes diabetes in experimental animals. ${ }^{3}$ Although nitrosoureas appear to function as bifunctional alkylating agents, the molecular mechanism of the function of the title compound has not been disclosed so far. To understand the molecular mechanism of the drug action, an X-ray analysis of the title compound was undertaken.

Suitable single crystals for X-ray diffraction experiments were obtained from an acetone solution. A colorless chip crystal having approximate dimensions of $0.25 \times 0.15 \times 0.10 \mathrm{~mm}$ was mounted on a glass fiber. The crystal and experimental data are given in Table 1.

The structure was solved by direct methods and refined by full-matrix least-squares methods with anisotropic temperature factors for non-H atoms. The absolute configuration of the molecule was suggested by referring to that of D-glucose. All H atoms which are bonded to carbon atoms were calculated geometrically and refined using the riding model. Other $\mathrm{H}$ atoms bonded to oxygen and nitrogen atoms were located from difference Fourier syntheses. These $\mathrm{H}$ atoms were refined with isotropic temperature factors. The atomic parameters for non- $\mathrm{H}$ atoms are listed in Table 2.

There are two crystallographically independent molecules of streptozocin in an asymmetric unit. ORTEP $\mathrm{III}^{4}$ drawings of the molecules together with the atomic numbering are shown in Fig. 2. The two independent molecules adopt essentially similar

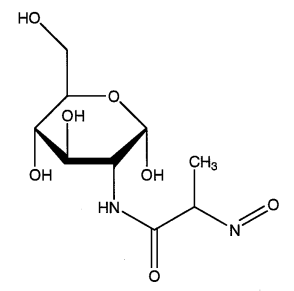

Fig. 1 Chemical structure of streptozocin.

\footnotetext{
$\doteqdot$ To whom correspondence should be addressed.

E-mail address: hirayama@is.icc.u-tokai.ac.jp
}

structures. Selected bond distances and angles are given in Table 3. The bond distances and angles are within the expected ranges. The bond angles around the O106-C107 and N102-N103 bonds and those around the O206-C207 and N202-N203 bonds are highly asymmetrical. The pyranose rings in both molecules take chair conformations. Although the hydroxyl groups at $\mathrm{C} 102$ and $\mathrm{C} 202$ are axial, all other functional groups attached to the pyranose ring are equatorial. Both of the nitrosourea groups are almost planar, but the orientations of these groups relative to the pyranose rings are different. In molecule $\mathrm{I}$, the torsion angles, C107-N101-C102-C103 and C107-N101-C102-C101, are $-169.7(6)$ and $68.4(8)^{\circ}$, respectively. The corresponding angles in molecule II, however, are $-146.3(6)$ and 90.0(7) ${ }^{\circ}$, respectively. In the crystal there is an extensive hydrogenbonding network. The geometrical details of the hydrogen bonds are tabulated in Table 4 . There are ten intermolecular hydrogen bonds and ten intramolecular hydrogen bonds. All potential hydrogen-bond donors and acceptors, except for N203, are engaged in the hydrogen bonds.

Table 1 Crystal and experimental data

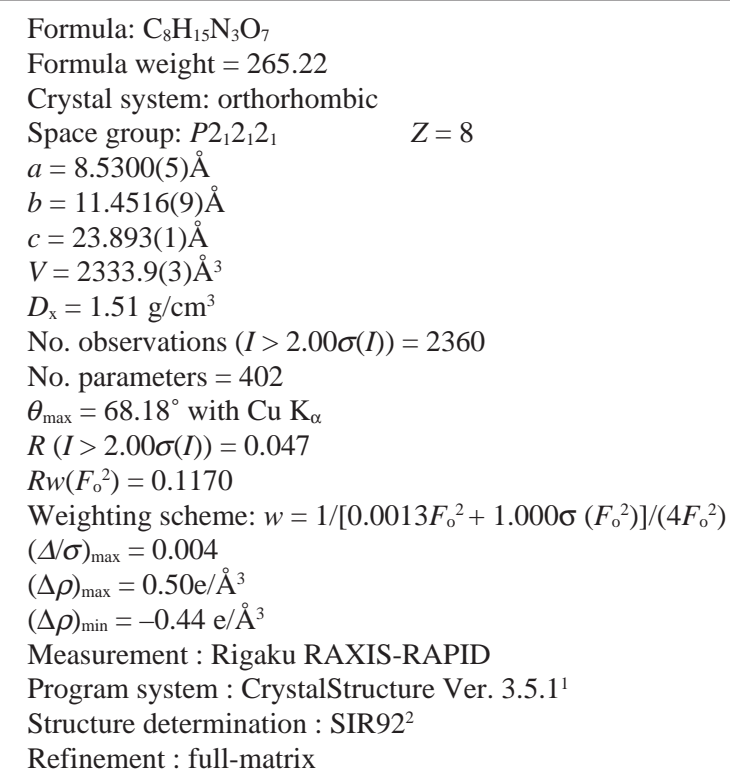


Table 2 Atomic coordinates and equivalent isotropic thermal parameters $\left(B_{\text {eq }}\right)$

\begin{tabular}{|c|c|c|c|c|}
\hline Atom & $x$ & $y$ & $z$ & $B_{\text {eq }}$ \\
\hline \multicolumn{5}{|c|}{ molecule I of streptozocin } \\
\hline 0101 & $0.6670(6)$ & $-0.2927(4)$ & $1.0764(2)$ & $3.2(1)$ \\
\hline $\mathrm{O} 102$ & $0.3909(6)$ & $-0.2309(4)$ & $1.2169(2)$ & 2.9(1) \\
\hline O103 & $0.5814(7)$ & $-0.4331(4)$ & $1.2444(2)$ & $3.0(1)$ \\
\hline $\mathrm{O} 104$ & $0.5201(5)$ & $-0.4616(4)$ & $1.0934(2)$ & $2.8(1)$ \\
\hline O105 & $0.4737(7)$ & $-0.6629(4)$ & $1.1782(2)$ & 4.1(1) \\
\hline O106 & $0.2753(6)$ & $-0.1728(4)$ & $1.0274(2)$ & $3.1(1)$ \\
\hline $\mathrm{O} 107$ & $0.3442(8)$ & $0.1795(4)$ & $1.0980(3)$ & $6.9(2)$ \\
\hline N101 & $0.4102(7)$ & $-0.1500(5)$ & $1.1074(2)$ & $2.4(1)$ \\
\hline N102 & $0.3136(6)$ & $0.0126(5)$ & $1.0598(2)$ & $2.9(1)$ \\
\hline N103 & $0.3673(8)$ & $0.0729(5)$ & $1.1025(3)$ & $4.8(2)$ \\
\hline C101 & $0.517(1)$ & $-0.3415(6)$ & $1.0785(3)$ & $2.7(2)$ \\
\hline C102 & $0.4204(9)$ & $-0.2735(6)$ & $1.1207(2)$ & $2.3(2)$ \\
\hline C103 & $0.4909(9)$ & $-0.2895(6)$ & $1.1784(3)$ & $2.3(2)$ \\
\hline C104 & $0.5070(9)$ & $-0.4182(5)$ & $1.1916(3)$ & $2.3(2)$ \\
\hline C105 & $0.596(1)$ & $-0.4819(6)$ & $1.1453(3)$ & $2.8(2)$ \\
\hline C106 & $0.611(1)$ & $-0.6112(6)$ & $1.1551(3)$ & $3.5(2)$ \\
\hline C107 & $0.3328(8)$ & $-0.1119(6)$ & $1.0638(3)$ & $2.4(1)$ \\
\hline C108 & $0.232(1)$ & $0.0662(7)$ & $1.0134(3)$ & $5.3(2)$ \\
\hline \multicolumn{5}{|c|}{ molecule II of streptozocin } \\
\hline O201 & $0.6205(6)$ & $-0.0308(4)$ & $1.1829(2)$ & $2.9(1)$ \\
\hline $\mathrm{O} 202$ & $1.0330(7)$ & $-0.1500(6)$ & $1.1057(3)$ & $5.5(2)$ \\
\hline O203 & $1.0694(7)$ & $-0.1840(5)$ & $1.2257(3)$ & $4.5(2)$ \\
\hline $\mathrm{O} 204$ & $0.8305(5)$ & $0.0786(3)$ & $1.2168(2)$ & $2.8(1)$ \\
\hline O205 & $1.1088(6)$ & $0.1146(5)$ & $1.2744(2)$ & $4.2(1)$ \\
\hline $\mathrm{O} 206$ & $0.7940(8)$ & $0.1538(5)$ & $1.0378(2)$ & $6.5(2)$ \\
\hline $\mathrm{O} 207$ & $0.5451(6)$ & $-0.1092(5)$ & $0.9365(2)$ & $4.6(1)$ \\
\hline N201 & $0.7543(7)$ & $-0.0273(5)$ & $1.0734(2)$ & $3.0(1)$ \\
\hline N202 & $0.6619(7)$ & $0.0222(5)$ & $0.9839(2)$ & $3.7(1)$ \\
\hline $\mathrm{N} 203$ & $0.6091(8)$ & $-0.0868(5)$ & $0.9806(2)$ & $3.5(1)$ \\
\hline C201 & $0.7383(8)$ & $0.0495(5)$ & $1.1698(3)$ & $2.6(2)$ \\
\hline $\mathrm{C} 202$ & $0.8407(8)$ & $-0.0020(5)$ & $1.1247(3)$ & $2.6(1)$ \\
\hline C203 & $0.9220(8)$ & $-0.1105(6)$ & $1.1465(3)$ & $3.0(2)$ \\
\hline C204 & $1.0111(8)$ & $-0.0808(6)$ & $1.1992(3)$ & $3.3(2)$ \\
\hline $\mathrm{C} 205$ & $0.9074(8)$ & $-0.0182(6)$ & $1.2410(3)$ & $2.9(2)$ \\
\hline C206 & $0.9970(9)$ & $0.0281(6)$ & $1.2903(3)$ & $3.9(2)$ \\
\hline $\mathrm{C} 207$ & $0.743(1)$ & $0.0550(7)$ & $1.0340(3)$ & $3.9(2)$ \\
\hline C208 & $0.646(1)$ & $0.1032(7)$ & $0.9379(3)$ & $6.9(3)$ \\
\hline
\end{tabular}

$B_{e q}=8 / 3 \pi^{2}\left(U_{11}\left(a a^{*}\right)^{2}+U_{22}\left(b b^{*}\right)^{2}+U^{33}\left(c c^{*}\right)^{2}+2 U_{12}\left(a a^{*} b b^{*}\right) \cos \gamma+\right.$ $\left.2 U_{13}\left(a a^{*} c c^{*}\right) \cos \beta+2 U_{23}\left(b b^{*} c c^{*}\right) \cos \alpha\right)$
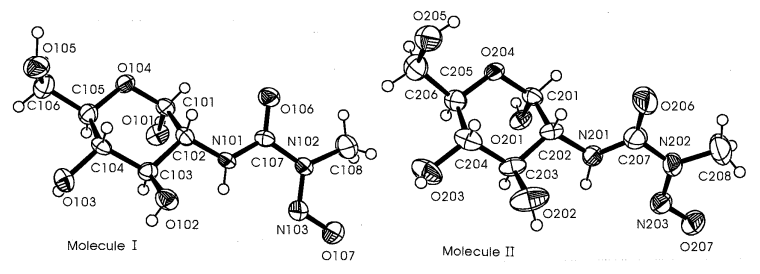

Fig. 2 Molecular structures of streptozocin with atom labeling. Thermal ellipsoids of non- $\mathrm{H}$ atoms are drawn at the $50 \%$ probability level.

\section{Acknowledgements}

This work was supported by Grants for the Key Technology Research Promotion Program of New Energy and Industrial Technology Development Organization (NEDO) of Japan, and also by the Research and Study Program of Tokai University Educational System General Research Organization.

\section{References}

1. CrystalStructure, version 3.5.1, 2000 - 2003 Crystal
Table 3 Selected bond lengths $(\AA)$ and angles $\left({ }^{\circ}\right)$

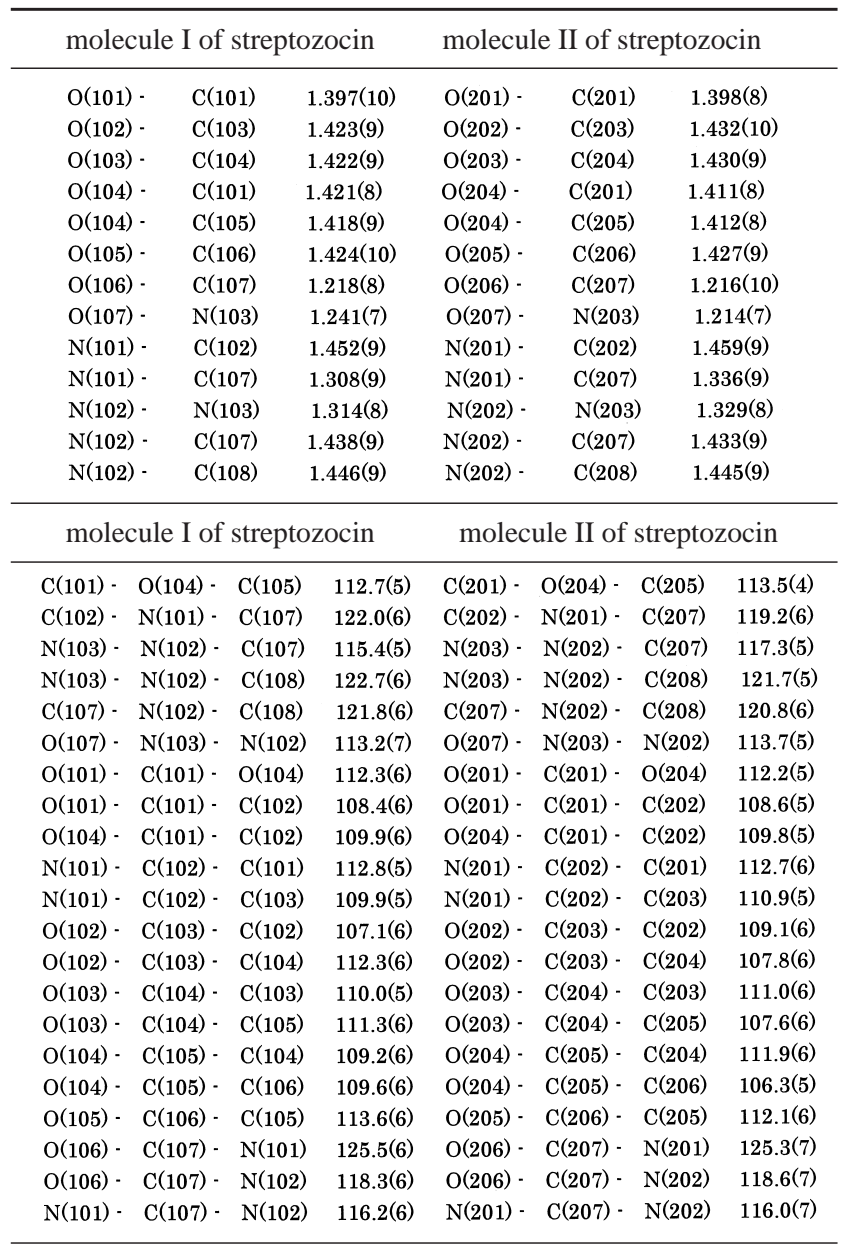

Table 4 Hydrogen bonds. A and D denote the hydrogen-bond acceptor and donor, respectively.

\begin{tabular}{|c|c|c|c|c|}
\hline $\mathrm{D}-\mathrm{H} \cdots \mathrm{A}$ & $\mathrm{D} \cdot \mathrm{H}(\AA)$ & $\mathrm{H} \cdots \mathrm{A}(\AA)$ & $\mathrm{D} \cdots \mathrm{A}(\AA)$ & $\angle \mathrm{D}-\mathrm{H}^{\cdots}-\mathrm{A}\left(^{\circ}\right)$ \\
\hline $\mathrm{O}(105) \cdot \mathrm{H}(4) \cdots \mathrm{O}(107)^{\mathrm{i}}$ & $0.96(9)$ & $1.91(9)$ & $2.855(8)$ & $170(8)$ \\
\hline $\mathrm{O}(203) \cdot \mathrm{H}(14) \cdots \mathrm{O}(102)^{\mathrm{ii}}$ & $0.81(9)$ & $2.00(9)$ & $2.802(8)$ & $168(9)$ \\
\hline $\mathrm{O}(101) \cdot \mathrm{H}(15) \cdots \mathrm{O}(106)^{\mathrm{iii}}$ & $0.85(7)$ & $1.84(7)$ & $2.676(7)$ & $170(7)$ \\
\hline $\mathrm{O}(202) \cdot \mathrm{H}(16) \cdots \mathrm{O}(207)^{\mathrm{iii}}$ & $0.75(9)$ & $2.26(9)$ & $2.938(9)$ & $151(9)$ \\
\hline $\mathrm{O}(102) \cdot \mathrm{H}(22) \cdots \mathrm{O}(105)^{\mathrm{iv}}$ & $0.71(7)$ & $2.18(7)$ & $2.867(7)$ & $163(7)$ \\
\hline $\mathrm{O}(201) \cdot \mathrm{H}(25) \cdots \mathrm{O}(103)^{\mathrm{iv}}$ & $0.89(6)$ & $1.81(5)$ & $2.690(7)$ & $168(5)$ \\
\hline $\mathrm{O}(205) \cdot \mathrm{H}(26) \cdots \mathrm{O}(203)^{\mathrm{v}}$ & $0.84(7)$ & $2.04(7)$ & $2.762(8)$ & $144(7)$ \\
\hline $\mathrm{O}(103) \cdot \mathrm{H}(29) \cdots \mathrm{O}(205)^{\mathrm{vi}}$ & $0.69(9)$ & $2.05(8)$ & $2.736(8)$ & $173(8)$ \\
\hline $\mathrm{C}(208) \cdot \mathrm{H}(19) \cdots \mathrm{O}(107)^{\mathrm{vii}}$ & $0.952(11)$ & $2.377(10)$ & $3.128(10)$ & $135.6(7)$ \\
\hline $\mathrm{C}(108) \cdot \mathrm{H}(27) \cdots \mathrm{O}(104)^{\mathrm{viii}}$ & $0.950(11)$ & $2.534(9)$ & $3.349(9)$ & $143.9(8)$ \\
\hline $\mathrm{N}(201) \cdot \mathrm{H}(9) \cdots \mathrm{O}(101)$ & $1.04(7)$ & $2.14(7)$ & $3.130(7)$ & $159(6)$ \\
\hline $\mathrm{N}(101) \cdot \mathrm{H}(12) \cdots \mathrm{O}(201)$ & $0.85(5)$ & $2.06(6)$ & $2.887(7)$ & $165(6)$ \\
\hline $\mathrm{C}(103) \cdot \mathrm{H}(11) \cdots \mathrm{O}(201)$ & $0.91(6)$ & $2.60(5)$ & $3.164(8)$ & $121(4)$ \\
\hline $\mathrm{N}(101) \cdot \mathrm{H}(12) \cdots \mathrm{N}(103)$ & $0.85(5)$ & $2.30(6)$ & $2.581(8)$ & $100(4)$ \\
\hline $\mathrm{O}(205) \cdot \mathrm{H}(26) \cdots \mathrm{O}(204)$ & $0.84(7)$ & $2.44(7)$ & $2.775(7)$ & $105(5)$ \\
\hline $\mathrm{C}(105)-\mathrm{H}(7) \quad \cdots \mathrm{O}(101)$ & $0.91(7)$ & $2.38(5)$ & $2.788(8)$ & $107(4)$ \\
\hline $\mathrm{C}(103) \cdot \mathrm{H}(11) \cdots \mathrm{O}(101)$ & $0.91(6)$ & $2.53(5)$ & $2.863(9)$ & $102(3)$ \\
\hline $\mathrm{C}(101) \cdot \mathrm{H}(17) \cdots \mathrm{O}(106)$ & $0.84(7)$ & $2.57(6)$ & $3.078(9)$ & $120(5)$ \\
\hline $\mathrm{C}(208) \cdot \mathrm{H}(19) \cdots \mathrm{O}(206)$ & $0.95(1)$ & $2.325(9)$ & $2.762(9)$ & $107.3(7)$ \\
\hline $\mathrm{C}(108) \cdot \mathrm{H}(27) \cdots \mathrm{O}(106)$ & $0.95(1)$ & $2.360(9)$ & $2.782(9)$ & $106.4(7)$ \\
\hline
\end{tabular}

symmetry code: (i) $\mathrm{x},-1+\mathrm{y}, \mathrm{z}$ (ii) $1+\mathrm{x}, \mathrm{y}, \mathrm{z}$ (iii) $1 / 2+\mathrm{x},-1 / 2-\mathrm{y}$, $2-\mathrm{z}$ (iv) $1-\mathrm{x}, 1 / 2+\mathrm{y}, 5 / 2-\mathrm{z}$ (v) $2-\mathrm{x}, 1 / 2+\mathrm{y}, 5 / 2-\mathrm{z}$ (vi) $2-\mathrm{x},-$ $1 / 2+y, 5 / 2-z$ (vii) $1 / 2+x, 1 / 2-y, 2-z($ viii) $-1 / 2+x,-1 / 2-y, 2-z$

Structure Analysis Package, Rigaku and Rigaku/MSC

2. SIR92 A. Altomare, G. Cascarano, C. Giacovazzo, A. Guagliardi, M. Burla, G. Polidori, and M. Camalli, J. Appl. Cryst., 1994, 27, 435.

3. "Goodman \& Gilman's The Pharmacological Basis of Therapeutics," ed. J. G. Hardman and L. E. Limbird, 10th ed., 2001, McGraw-Hill, New York, 1398.

4. ORTEP III L. J. Farrugia, J. Appl. Cryst., 1997, 22, 389 